\title{
Energy Allocation for Short-Packet Massive Multiple Access with Two-Iteration Successive Decoding
}

\author{
Francesc Molina and Josep Sala-Álvarez \\ Signal Theory and Communications Department, Technical University of Catalonia (UPC) \\ E-mail: \{francesc.molina, josep.sala $\} @$ upc.edu
}

\begin{abstract}
An optimum energy allocation for massive user networks operating under iterative successive interference cancellation (I-SIC) is derived in this work. We analyze an uplink setting where a large network of cost-effective low-power users employ a practical transmission scheme consisting of the same channel encoder followed by spread spectrum modulation. Variational calculus (VC) is utilized to derive the asymptotic large-user energy distribution that favors I-SIC's performance, in terms of spectral efficiency (SE), when at most two decoding attempts per user are performed. The capacity region under random spreading and SIC operating in the infinite blocklength regime is evaluated and contrasted with the maximized SE using short packets.

Index Terms-Massive multiple access, iterative successive interference cancellation, energy allocation, variational calculus.
\end{abstract}

\section{INTRODUCTION}

Multiple access (MA) interference constitutes one of the impairments to increasing the SE of future wireless networks [1], [2]. IC receivers have proved relevant in achieving substantial MA interference reductions. In fact, SIC attains the maximum sum-rate points of the Gaussian MA channel capacity region at a reasonable computation complexity through a successive interplay between decoding and cancellation of users [3]-[5].

The massive MA context entails non-ideal SIC operation owing to the fact that users transmit short rather than asymptotically long packets [6]. Hence, since channel decoders are nonerror free, some users may be incorrectly decoded during the SIC procedure. Thus, SIC performance is strongly determined by the packet error rate (PER) characteristic of the employed encoding and modulation system. The previous issue can be counteracted, firstly, by introducing error control to detect unsuccessfully decoded packets, and secondly, by iterating the SIC algorithm over users yet undecoded, namely I-SIC [7]. For system modeling, difficulty lies in capturing the statistical dependence between different decoding attempts of every user, which is obviated in [7]. In [8], a novel and accurate analysis to model each iteration of a large I-SIC system is proposed.

One critical aspect of SIC, though, is that user power levels must significantly differ to enable successful IC, and thus, the design of a proper energy allocation plays a vital role. In [9], SIC operating in the space-code domain is studied. In [10], energy allocation is designed for SIC under hybrid nonorthogonal MA from an energy efficiency point of view.

This work is supported in part by the Spanish Government through project TEC2016-76409-C2-1-R (AEI/FEDER, UE) (WINTER), in part by the Catalan administration (AGAUR) under Grant 2017 SGR 578, and in part by the Secretary for Universities and Research of the Generalitat de Catalunya and by the European Social Fund under Fellowship 2019FI_B1 00085.
Relevant works on satellite networks implementing Enhanced Spread Spectrum ALOHA (E-SSA) [7] have examined the direct application of I-SIC. Therein, a massive number of sporadic spread spectrum transmissions as described in [11] are analyzed under SIC operation. Several allocations have been designed for these settings in [12]-[14]. Theoretical analyses have shown that, when infinite blocklength codes are employed, users must transmit at the same rate and arrive with exponentially decaying energies to achieve the same SINR [12]. Slight differences are obtained in the finite blocklength regime for sufficiently large packets [13], [14]. Yet, the SE can still be increased by taking advantage of I-SIC [7], [8].

In this work, we adopt the E-SSA satellite framework, and analyze the user-asymptotic performance of a short-packet massive MA network under the same channel encoder and a twoiteration SIC, rather than the non-iterative SIC in [12]-[14]. The mathematical background in [8] is used to characterize every iteration of I-SIC under non-ideal channel decoding and imperfect cancellation. In particular, univariate and bivariate PER curves to model decoding in the first and second iterations are respectively adopted. We design the SE-optimum energy allocation under the assumption of having a large number of users at the beginning of each iteration and an accurate lower bound on I-SIC performance. We show that I-SIC reaps more benefits in terms of SE when using short packets because the second iteration better improves the decoding capabilities of less powerful encoding schemes. For abrupt PER curves, usually associated with longer packets, I-SIC performance is slightly improved with respect to that of a non-iterative SIC where users are decoded only in the first iteration.

For paper organization: Sec. II presents the system model. Sec. III derives network asymptotics. Sec. IV tackles optimization. Results are offered in Sec. V and conclusions in Sec. VI.

\section{SySTEM MODEL}

Consider $K$ users connected to the same destination node. Users generate packets by encoding data using the same channel encoder after which, transmission is performed under nonorthogonal MA and users superposed in the code domain by using spreading codes with period comprising many symbols.

At reception, a specific I-SIC strategy is performed to demodulate and decode the $K$ interfering packets. We simplify the iterative decoding procedure in [7], [8] opting for an I-SIC receiver that performs 2 iterations, actually the major contributors to characterizing the full I-SIC system performance. The

(C) 2020 IEEE. Personal use of this material is permitted. Permission from IEEE must be obtained for all other uses, in any current or future media, including reprinting/republishing this material for advertising or promotional purposes, creating new collective works, for resale or redistribution to servers or lists, or reuse of any copyrighted component of this work in other works. 
first iteration consists of $K_{1}=K$ demodulation and decoding stages whereas the second iteration provides a new decoding attempt to those unsuccessfully decoded users.

We focus our analysis in environments where user-channel gains are practically stationary over the packet duration so that, a SINR-based system model after symbol despreading together with the Gaussian interference assumption supported by the use of long spreading codes, provides an accurate, useful and simplified system model for I-SIC's behavior.

\section{A. SIC Operation: The First Iteration}

At the first SIC iteration, user packets are ranked in nonincreasing order of received symbol energies $E_{\mathrm{r}}^{1}\left[1 \leq k_{1} \leq K_{1}\right]$, with the user index $1 \leq k_{1} \leq K_{1}$ defined to that purpose. At stage $k_{1}$ out of $K_{1}$, the spreading waveform of user $k_{1}$ is used as symbol-matched filter so that, the $k_{1}$-th user's SINR reads

$$
\Gamma^{1}\left[k_{1}\right]=\frac{E_{\mathrm{r}}^{1}\left[k_{1}\right]}{N_{t}^{1}\left[k_{1}\right]}=\frac{E_{\mathrm{r}}^{1}\left[k_{1}\right]}{N_{0}+\frac{\theta}{N_{i<k_{1}}} \sum_{1} \epsilon_{1}[i] E_{\mathrm{r}}^{1}[i]+\frac{\theta}{N_{i>k_{1}}} \sum_{\mathrm{r}} E^{1}[i]} .
$$

$N_{t}^{1}\left[k_{1}\right]$ contains the noise power spectral density $N_{0}$ plus the interference from processed $\left(i<k_{1}\right)$ and remaining $\left(i>k_{1}\right)$ users. The factor $\frac{\theta}{N}$ is the inter-user decorrelation [11]. To account for imperfect decoding-cancellation the random variable $\epsilon_{1}$ is introduced, whose distribution depends on the known PER vs. SINR curve $\operatorname{PER}_{1}\left[\Gamma^{1}\right] . \epsilon_{1}$ equals 1 with probability $\mathrm{PER}_{1}\left[\Gamma^{1}\right]$ (decoding error $\equiv$ no cancellation) or $0 \leq \varepsilon\left(\Gamma^{1}\right)<1$ (residual energy fraction of canceled users) otherwise.

\section{B. SIC Operation: The Second Iteration}

After the first iteration, $K_{2}$ users have been unsuccessfully decoded, and they undergo a second decoding attempt. This is the reason that has motivated us to define: (i) the new user index $1 \leq k_{2} \leq K_{2}$; and (ii) the mapping $\varphi^{-1}: k_{2} \rightarrow k_{1}$ to obtain the index of user $k_{2}$ in the first user index as $k_{1}=$ $\varphi^{-1}\left[k_{2}\right]$ [8]. Then, the new user-energy distribution is

$$
E_{\mathrm{r}}^{2}\left[k_{2}\right]=E_{\mathrm{r}}^{1}\left[\varphi^{-1}\left[k_{2}\right]\right] .
$$

In the second iteration, the channel decoder operates with the same signal as in the first iteration minus that of already canceled users. Thus, both decoding attempts are statistically dependent. Next, we exemplify the former dependence by analyzing the proof-of-concept situation where a user with SINR $\Gamma^{2}$, referred to as target user, undergoes a second decoding attempt after packet error at SINR $\Gamma^{1} \leq \Gamma^{2}$ occurred in the first iteration. Two cases are analyzed:

1) If no users have been canceled between iterations, the target user does not improve its SINR. That is, $\Gamma^{1}=\Gamma^{2}$, and a packet error occurs again since the channel decoder applies a deterministic function to the observation.

2) If a large number of cancellations is performed, the target user improves its SINR sufficiently so as to assume that both decoding attempts are statistically independent.

In this work, we adopt analogously to [8], a general form to analyze the statistical dependence between decoding attempts of both iterations as a function of the SINRs at every iteration,
$\Gamma^{1}$ and $\Gamma^{2}$. This allows us to continue characterizing the I-SIC under a SINR-based model and to include statistical dependencies between iterations as regards channel decoding. Specifically, to capture the soft behavior of channel decoding between a null and a high SINR improvement, we adopt the bivariate $\mathrm{PER}_{2}\left[\Gamma^{1}, \Gamma^{2}\right]$ to denote the PER of a user unsuccessfully decoded in the first iteration under SINR $\Gamma^{1}$ and having SINR $\Gamma^{2}$ in the second iteration.

The SINR of the $k_{2}$-th user $\Gamma^{2}\left[k_{2}\right] \triangleq E_{\mathrm{r}}^{2}\left[k_{2}\right] / N_{t}^{2}\left[k_{2}\right]$ is

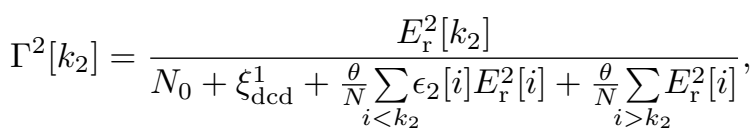

with $\xi_{\text {dcd }}^{1}$ the interference from the successfully decoded users in the first SIC iteration, $\epsilon_{2} \equiv \epsilon_{2}\left(\Gamma^{2}\right)$ a random variable defined analogously to $\epsilon_{1}$ using $\operatorname{PER}_{2}\left[\boldsymbol{\Gamma}^{2}\right]$ and $\varepsilon\left(\Gamma^{2}\right)$, and $\Gamma^{2} \triangleq\left[\Gamma^{1}, \Gamma^{2}\right]$ the vector of SINR values a given user experiences. In particular, for the $k_{2}$-th user in the second iteration:

$$
\Gamma^{2}\left[k_{2}\right] \triangleq\left[\Gamma^{1}\left[\varphi^{-1}\left[k_{2}\right]\right], \Gamma^{2}\left[k_{2}\right]\right],
$$

where $k_{1}=\varphi^{-1}\left[k_{2}\right]$ gets the index of user $k_{2}$ in $1 \leq k_{1} \leq K_{1}$.

\section{USER-LIMIT ANALYSIS}

The asymptotic system where both the number of users $K$ and the spreading gain $N$ tend to infinity [12] is analyzed in this section. The asymptotic user indices $t_{1 \leq i \leq 2} \triangleq$ $\lim _{K_{i} \rightarrow+\infty} k_{i} / K_{i}$ are defined, and the traffic loads at each SIC iteration $\alpha_{1 \leq i \leq 2} \triangleq K_{i} / N$ are held fixed. Naturally, the assumption of having a large number of users after the first SIC iteration is subject to having $K_{2}=\alpha_{2} \cdot N$ sufficiently high. In fact, the real system highlights a combination between large $K_{1}$ and moderate/low $K_{2}$, that we solve by letting both go to infinity, which has been proved true in [8] for some examples. Hence, under the user-limit system model, the functions under the user-indices $k_{1}, k_{2}$ in Section II are turned to asymptotic functions of the user-indices $t_{1}, t_{2}$ now denoted profiles. For instance, the energy profiles are $E_{\mathrm{r}}^{i}\left[K_{i} t_{i}\right] \rightarrow E_{\mathrm{r}}^{i}\left(t_{i}\right)$.

In this user-limit situation, after the first iteration, every interval of users with length $\mathrm{d} t_{2}$ consists of a fraction $\mathrm{PER}_{1}\left[\Gamma^{1}\left(t_{1}\right)\right] \mathrm{d} t_{1}$ of undecoded users. This is,

$$
\mathrm{d} t_{2}=\operatorname{PER}_{1}\left[\Gamma^{1}\left(t_{1}\right)\right] \mathrm{d} t_{1} .
$$

Now, integrating both terms, the indexing $\varphi$ introduced in Section II-B is turned to the bijection $t_{2}=\varphi\left(t_{1}\right)$ in (6), where the denominator is introduced as a normalization term so that $\varphi$ defines a mapping from $t_{1} \in[0,1]$ to $t_{2} \in[0,1]$ :

$$
t_{2}=\varphi\left(t_{1}\right)=\int_{0}^{t_{1}} \operatorname{PER}_{1}\left[\Gamma^{1}(\tau)\right] \mathrm{d} \tau / \int_{0}^{1} \operatorname{PER}_{1}\left[\Gamma^{1}(\tau)\right] \mathrm{d} \tau \text {. }
$$

An analytic expression for the asymptotic energy distribution of users that survive after the first iteration (2) is obtained:

$$
E_{\mathrm{r}}^{2}\left(t_{2}\right)=E_{\mathrm{r}}^{1}\left(\varphi^{-1}\left(t_{2}\right)\right) \text {. }
$$

The interesting result obtained herein is that, instead of (2) which depends on whether channel decoding is successful or not, $E_{\mathrm{r}}^{2}\left(t_{2}\right)$ in (7) can be computed analytically using (6).

Under this asymptotic large-user assumption the system model in Section II can be turned to deterministic expressions. 


\section{A. User-Asymptotic SINR Profiles}

The previous user-SINR expressions (1) and (3) are turned to their asymptotic profiles $\Gamma^{1 \leq i \leq 2}\left(t_{i}\right)=E_{\mathrm{r}}^{i}\left(t_{i}\right) / N_{t}^{i}\left(t_{i}\right)$ as

$$
\Gamma^{i}\left(t_{i}\right)=\frac{E_{\mathrm{r}}^{i}\left(t_{i}\right)}{N_{0}^{i}+\alpha_{i} \theta \int_{0}^{t_{i}} r_{i}(\tau) E_{\mathrm{r}}^{i}(\tau) \mathrm{d} \tau+\alpha_{i} \theta \int_{t_{i}}^{1} E_{\mathrm{r}}^{i}(\tau) \mathrm{d} \tau},
$$

with $N_{0}^{1} \triangleq N_{0}$ and $N_{0}^{2} \triangleq N_{0}+\xi_{\text {dcd }}^{1}$ the initial noise terms, and $r_{i} \triangleq 1-\left(1-\varepsilon\left(\Gamma^{i}\right)\right) \operatorname{PSR}_{i}\left[\boldsymbol{\Gamma}^{i}\right]$ the expectation of $\epsilon_{i}$.

\section{B. Noise Plus Interference Profiles}

As stated in [8], differentiating and manipulating terms, two differential equations are obtained for $i=\{1,2\}$ :

$$
\nabla_{t_{i}} \log N_{t}^{i}\left(t_{i}\right)=-\alpha_{i} \Phi_{i}\left[\boldsymbol{\Gamma}^{i}\left(t_{i}\right)\right],
$$

with $\Phi_{i}\left[\boldsymbol{\Gamma}^{i}\right] \triangleq \theta\left(1-\varepsilon\left(\Gamma^{i}\right)\right) \Gamma^{i} \cdot \operatorname{PSR}_{i}\left[\boldsymbol{\Gamma}^{i}\right]$ a known function. Therefore, by solving the previous ordinary differential equations the noise plus interference profiles associated with every iteration yield

$$
\begin{aligned}
& N_{t}^{1}\left(t_{1}\right)=N_{t}^{1}(0) \exp \left(-\alpha_{1} \int_{0}^{t_{1}} \Phi_{1}\left[\Gamma^{1}(\tau)\right] \mathrm{d} \tau\right), \\
& N_{t}^{2}\left(t_{2}\right)=N_{t}^{1}(1) \exp \left(-\alpha_{2} \int_{0}^{t_{2}} \Phi_{2}\left[\boldsymbol{\Gamma}^{2}(\tau)\right] \mathrm{d} \tau\right),
\end{aligned}
$$

with $N_{t}^{1}(0) \triangleq N_{0}+\alpha_{1} \theta \int_{0}^{1} E_{\mathrm{r}}^{1}(t) \mathrm{d} t$ and $N_{t}^{2}(0)=N_{t}^{1}(1) \triangleq$ $N_{0}+\xi_{\text {dcd }}^{1}$ the boundary noise terms, $\alpha_{2}=\alpha_{1} \overline{\operatorname{per}}_{1}$ the uncanceled load after the first iteration, and (4) turned to

$$
\Gamma^{2}\left(t_{2}\right)=\left[\Gamma^{1}\left(\varphi^{-1}\left(t_{2}\right)\right), \Gamma^{2}\left(t_{2}\right)\right] .
$$

\section{Network Figure of Merit: The Average PER}

We define one figure of merit at the end of every iteration, and a global utility accounting for the total network performance after both iterations. The latter is closely related with the user-aggregate spectral efficiency

$$
\mathrm{SE}=\alpha_{1} R \cdot(1-\overline{\mathrm{per}}) .
$$

$\alpha_{1} R$ is the aggregate number of information bits per unit bandwidth transmitted by all users, and $\overline{\text { per }}$ is the global utility that accounts for the long-term average number of erroneously decoded packets from the network perspective detailed below.

The average fraction of unsuccessfully decoded users after computing iterations 1 and 2 individually, referred to as partial PERs, are asymptotically computed as

$$
\begin{aligned}
& \overline{\operatorname{per}}_{1}=\int_{0}^{1} \operatorname{PER}_{1}\left[\Gamma^{1}\left(t_{1}\right)\right] \mathrm{d} t_{1}, \\
& \overline{\operatorname{per}}_{2}=\int_{0}^{1} \operatorname{PER}_{2}\left[\Gamma^{2}\left(t_{2}\right)\right] \mathrm{d} t_{2},
\end{aligned}
$$

and the average fraction of unsuccessfully decoded users after two SIC iterations, denoted herein average PER, factorizes as the product of the partial PERs in (14)-(15):

$$
\begin{aligned}
\overline{\mathrm{per}} & =\overline{\mathrm{per}}_{1} \cdot \overline{\mathrm{per}}_{2} \\
& =\int_{0}^{1} \operatorname{PER}_{1}\left[\Gamma^{1}\left(t_{1}\right)\right] \mathrm{d} t_{1} \int_{0}^{1} \operatorname{PER}_{2}\left[\boldsymbol{\Gamma}^{2}\left(t_{2}\right)\right] \mathrm{d} t_{2} .
\end{aligned}
$$

The former user-asymptotic system model is used henceforth to formulate and solve an optimization problem.

\section{Asymptotic Energy Allocation Design}

In this section, we design such energy allocation to attain the most favorable user-energy distribution to SIC's performance measured in terms of the network SE. Accordingly, the

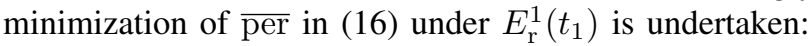

$$
\begin{aligned}
\min _{E_{\mathrm{r}}^{1}\left(t_{1}\right)} & \overline{\mathrm{per}} \\
\text { s.t. } & \bar{E}=\int_{0}^{1} E_{\mathrm{r}}^{1}\left(t_{1}\right) \mathrm{d} t_{1} \\
\text { s.t. } & E_{\mathrm{r}}^{1}\left(t_{1}\right)=\Gamma^{1}\left(t_{1}\right) N_{t}^{1}\left(t_{1}\right) \\
\text { s.t. } & E_{\mathrm{r}}^{2}\left(t_{2}\right)=\Gamma^{2}\left(t_{2}\right) N_{t}^{2}\left(t_{2}\right) \\
\text { s.t. } & E_{\mathrm{r}}^{1}\left(t_{1}\right)=E_{\mathrm{r}}^{2}\left(\varphi\left(t_{1}\right)\right)
\end{aligned}
$$

and subject to: (i) an average energy constraint over the user population (18) (a reasonable constraint to limit energy expenditure as in [13], [14]); (ii) the equations of the adopted SIC algorithm in the first (19) and second (20) SIC iterations with the noise plus interference profiles $N_{t}^{1}\left(t_{1}\right)$ and $N_{t}^{2}\left(t_{2}\right)$ in (10)-(11); and (iii) the asymptotic relationship between energy profiles of both iterations in (7) with $t_{2}=\varphi\left(t_{1}\right)$.

\section{A. A Conservative Energy Allocation Design}

In the sequel, we make some considerations to simplify the former problem. In fact, there are two main reasons why we are interested in simplifying $\mathrm{PER}_{2}\left[\Gamma^{1}, \Gamma^{2}\right]$. Firstly, it is easy to see that the solution will not only depend on $\mathrm{PER}_{2}$ itself, but also on their partial derivatives, which are, in general, difficult to obtain. Secondly, a robust energy allocation under a conservative hypothesis may have more practical interest, rather than the exact SIC behavior. Consequently, instead of adopting the true two-variate $\operatorname{PER}_{2}\left[\Gamma^{1}, \Gamma^{2}\right]$, the following upper-bound first derived in [8] is considered:

$$
\operatorname{PER}_{1}\left[\Gamma^{1}\right] \mathrm{PER}_{2}\left[\Gamma^{1}, \Gamma^{2}\right] \leq \mathrm{PER}_{1}\left[\Gamma^{2}\right] .
$$

It is shown therein that the former is very tight as the ratio $\Gamma^{2} / \Gamma^{1}$ increases. Hence, we solve for $\mathrm{PER}_{2}$ in (22) and take the result as a strict equality (with validity at $\Gamma^{2} \geq \Gamma^{1}$ ):

$$
\mathrm{PER}_{2}\left[\Gamma^{1}, \Gamma^{2}\right]=\operatorname{PER}_{1}\left[\Gamma^{2}\right] / \mathrm{PER}_{1}\left[\Gamma^{1}\right] .
$$

Reasonable insight about SIC operation can be extracted from (23). The underlying idea is that the second SIC iteration is useful as long as users improve significantly their SINRs between iterations. The performance of channel decoding in the second SIC iteration is given by the ratio of univariate PERs evaluated at the SINRs $\Gamma^{2}$ and $\Gamma^{1}$. Moreover, a mathematical justification for $\mathrm{PER}_{2}$ in (23) follows in the footnote ${ }^{1}$.

\footnotetext{
${ }^{1}$ We check in the sequel that $\mathrm{PER}_{2}$ verifies the properties of any bivariate PER function by formulating two axioms over the univariate $\mathrm{PER}_{1}[\Gamma]$ : (i) bounded in $[0,1]$; and (ii) non-increasing in $\Gamma$. These axioms are actually fulfilled by any PER characteristic (see some examples in Section V). Hence:

(a) Property 1: $\operatorname{PER}_{2}\left[\Gamma^{1}, \Gamma^{2}\right] \in[0,1]$ since $0<\Gamma^{1} \leq \Gamma^{2}<\infty$.

(b) Property 2: Non-increasing in $\Gamma^{2}$. Proof: $\partial_{\Gamma^{2}} \mathrm{PER}_{2}=\mathrm{PER}_{1}^{\prime}\left[\Gamma^{2}\right] / \mathrm{PER}_{1}\left[\Gamma^{1}\right] \leq 0$ since $\mathrm{PER}_{1}^{\prime} \leq 0$.

(c) Property 3: Non-decreasing in $\Gamma^{1}$ Proof: $\partial_{\Gamma^{1}} \mathrm{PER}_{2}=-\mathrm{PER}_{2}\left[\Gamma^{1}, \Gamma^{2}\right] \mathrm{PER}_{1}^{\prime}\left[\Gamma^{2}\right] / \mathrm{PER}_{1}\left[\Gamma^{2}\right] \geq 0$.

(d) Property 4: $\mathrm{PER}_{2}\left[\Gamma^{1}, \Gamma^{2}\right] \rightarrow 1$ when $\Gamma^{2} \rightarrow \Gamma^{1}$, that is, the decoder does not succeed under poor SINR gains.
} 


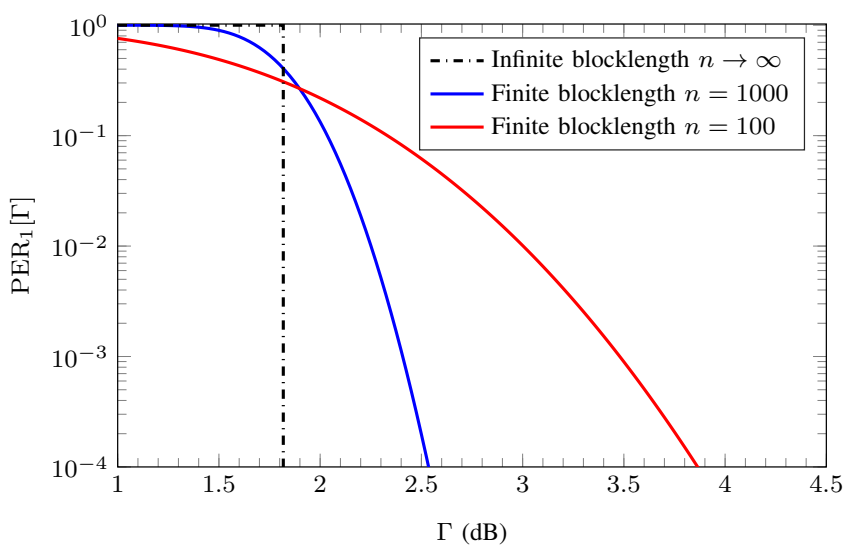

Fig. 1. PER characteristic $\mathrm{PER}_{1}[\Gamma]$ vs. $\Gamma(\mathrm{dB})$ for a number of blocklengths $n$ and $R=4 / 3$. The PER characteristic of the capacity-achieving code with cutoff SINR $2^{R}-1=1.82 \mathrm{~dB}$ is depicted using a dash-dotted line, and that associated with the blocklengths 100 and 1000 using solid lines.

Therefore, the following lines are devoted to analyzing the previous system expressions when (23) is verified:

1) Average PER: The change of variable $t_{2} \triangleq \varphi\left(t_{1}\right)$ with $\mathrm{d} t_{2}=\varphi^{\prime}\left(t_{1}\right) \mathrm{d} t_{1}=\operatorname{PER}_{1}\left[\Gamma^{1}\left(t_{1}\right)\right] / \overline{\operatorname{per}}_{1} \mathrm{~d} t_{1}$ is applied over (16), which now becomes

$$
\overline{\mathrm{per}}=\int_{0}^{1} \operatorname{PER}_{1}\left[\Gamma^{2}\left(\varphi\left(t_{1}\right)\right)\right] \mathrm{d} t_{1} .
$$

2) Noise plus interference term: Applying the same change of variable to $N_{t}^{2}\left(t_{2}\right)$ in (11) and after some straightforward manipulations, we get

$$
N_{t}^{2}\left(t_{2}\right)=N_{t}^{1}(1) \exp \left(-\alpha_{1} \int_{0}^{\varphi^{-1}\left(t_{2}\right)} \Psi\left[\Gamma^{1}(\tau), \Gamma^{2}(\varphi(\tau))\right] \mathrm{d} \tau\right)
$$

with $\Psi\left[\Gamma^{1}, \Gamma^{2}\right] \triangleq \theta\left(1-\varepsilon\left(\Gamma^{2}\right)\right) \Gamma^{2}\left(\operatorname{PER}\left[\Gamma^{1}\right]-\operatorname{PER}\left[\Gamma^{2}\right]\right)$.

\section{B. The Optimum Energy Allocation Design}

We design $E_{\mathrm{r}}^{1}\left(t_{1}\right)$ in $0 \leq t_{1} \leq t_{*}$, with $0<t_{*} \leq 1$ a user index that sets $E_{\mathrm{r}}^{1}\left(t_{1}\right)=0$ in $t_{*}<t_{1} \leq 1$. Hence, we turn (17)-(21) to an equivalent optimization under $\Gamma^{1}\left(t_{1}\right)$ and $\Gamma^{2}\left(\varphi\left(t_{1}\right)\right)$ profiles by substituting: firstly, (19) into (18) and (21); and secondly, (20) (with $t_{2}=\varphi\left(t_{1}\right)$ ) into (21). Then:

$$
\begin{aligned}
\min _{0<t_{*} \leq 1} \min _{\Gamma^{1}(t), \Gamma^{2}(\varphi(t))} \int_{0}^{t_{*}} \operatorname{PER}_{1} & {\left[\Gamma^{2}(\varphi(t))\right] \mathrm{d} t } \\
\text { s.t. } & \bar{E}=\int_{0}^{t_{*}} \Gamma^{1}(t) N_{t}^{1}(t) \mathrm{d} t \\
\text { s.t. } \quad \Gamma^{1}(t) N_{t}^{1}(t) & =\Gamma^{2}(\varphi(t)) N_{t}^{2}(\varphi(t))
\end{aligned}
$$

The former constitutes, firstly, two VC problems under the continuously differentiable $\Gamma^{1}(t), \Gamma^{2}(\varphi(t))$ in $0 \leq t \leq t_{*}$, where for readability we have substituted $t_{1}$ by $t$, and secondly, the scalar optimization of $0<t_{*} \leq 1$. The inner problem can be solved analytically using variational calculus tools. The explicit derivation has been translated to the Appendix, where the stationary point profiles are shown to be those verifying the following in $0 \leq t \leq t_{*}$

$$
\lambda=\frac{\operatorname{PER}_{1}^{\prime}\left[\Gamma_{0}^{2}(\varphi(t))\right]}{\beta(t) N_{t}^{2}(\varphi(t))-\alpha_{1} I_{\mathrm{x}}(t) \Psi_{\Gamma^{2}}\left[\boldsymbol{\Gamma}_{0}^{2}(\varphi(t))\right]},
$$

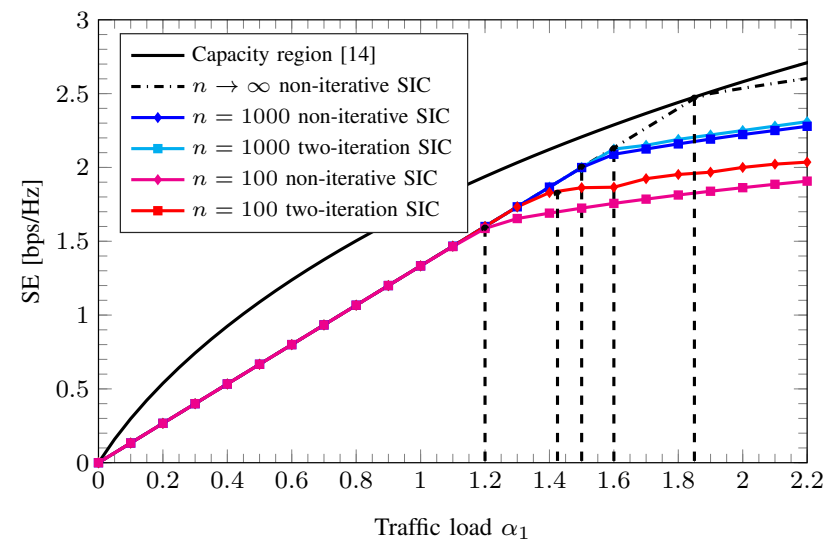

Fig. 2. SE vs. traffic load $\alpha_{1}$. The optimum SE under a non-iterative SIC is depicted using squares, whereas the two-iteration SIC is shown in diamond. Dashed lines indicate the limit traffic loads at which $t_{*}=1$.

with $I_{\mathrm{x}}(t) \triangleq \int_{t}^{t_{*}} \beta(\tau) E_{\mathrm{r}}^{1}(\tau) \mathrm{d} \tau$, and $\beta(t)$ computed as

$$
\beta(t)=\alpha_{1} \frac{\tilde{I}(t) \Phi^{\prime}\left[\Gamma_{0}^{1}(t)\right]-I_{\mathrm{x}}(t) \Psi_{\Gamma^{1}}\left[\boldsymbol{\Gamma}_{0}^{2}(\varphi(t))\right]}{N_{t}^{1}(t)}-1,
$$

with $I(t) \triangleq \int_{t}^{t_{*}} E_{\mathrm{r}}^{1}(\tau) \mathrm{d} \tau$, and $\tilde{I}(t) \triangleq I(t)+I_{\mathrm{x}}(t)-I_{\mathrm{x}}(0)$.

\section{Simulation Results and Discussion}

We compare the I-SIC performance for three optimum encoding schemes in terms of the maximal channel coding rate at infinite/finite blocklength [6]. We are interested in evaluating the impact of the PER curve's abruptness on SE. To that aim, we set the encoder's rate at $R=4 / 3 \mathrm{bps} / \mathrm{Hz}$ and evaluate the packet lengths $n=\{100,1000,+\infty\}$. Their PER characteristics are depicted in Fig. 1. For simulations, the average symbol energy over the noise spectral density $N_{0}$ is $\bar{E} / N_{0}=10 \mathrm{~dB}$, and $\theta$ is set to 1 . The factor for uncanceled energy is assumed $\Gamma$-independent [13] with $\varepsilon(\Gamma)=0.01$.

The solution is obtained by solving (29)-(30) under a discrete vector $t$ of $10^{3}$ samples. We have found that our proposal is relevant for low/moderate activity loads. For increasing loads, high accuracy solvers are required to validate the obtained equations. In that case, we discretize the optimization (26)-(28) and obtain its solution numerically.

\section{A. Spectral Efficiency}

We show in Fig. 2 the SE vs. the system load $\alpha_{1}$ when transmitting infinite- or finite-length packets. In the first regime, since channel decoders are error-free all users can be decoded in the first SIC iteration. The capacity region (evaluated in [14]) states the optimum SINR profile is uniform and users transmit at the same rate selected as a function of $\alpha_{1}$. The SE using the capacity-achieving code in Fig. 2 is increasing in $\alpha_{1}$ and attains the capacity region at $\alpha_{1}=1.85$. In the short-packet regime, users are subject to a second decoding attempt if their first attempt does not succeed. We compare the optimized SE when optimizing a non-iterative SIC following [13] with a two-iteration SIC as proposed herein. We put emphasis on and analyze the limit traffic loads at which the user index $t_{*}$ is set to 1 , namely saturation loads (dashed). 


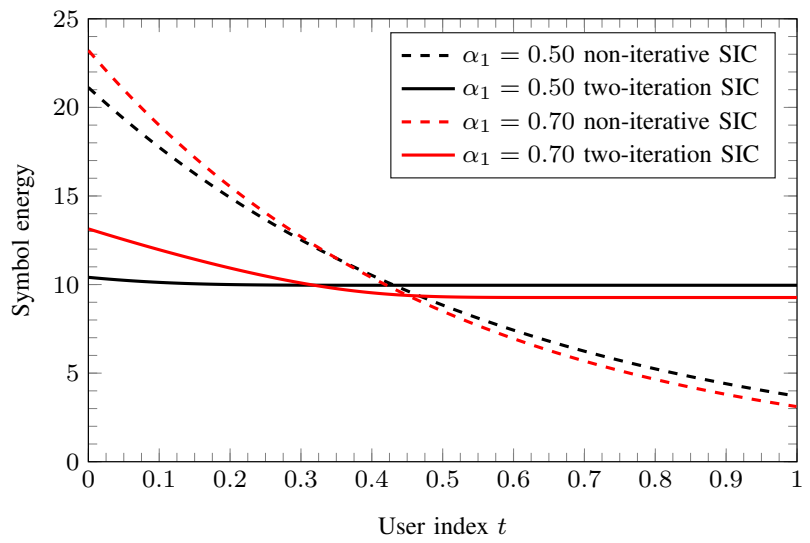

Fig. 3. Optimum energy profile $E_{\mathrm{r}}^{1}(t)$ when optimizing up to the first SIC iteration [13] (dashed) and up to the second SIC iteration (solid).

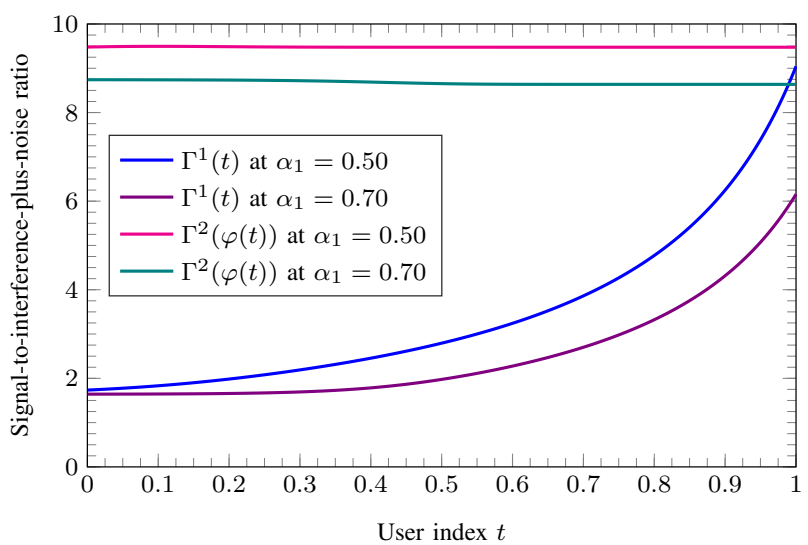

Fig. 4. SINR profiles associated with the first $\Gamma^{1}(t)$ and second $\Gamma^{2}(\varphi(t))$ iterations.

With regard to the non-iterative SIC, the saturation loads associated with the packet lengths 100 and 1000 are near 1.20 and 1.50 , and the SEs 1.60 and $2.00 \mathrm{bps} / \mathrm{Hz}$ are achieved. The former SEs can be increased further when using a twoiteration SIC, viz: 1.83 and $2.13 \mathrm{bps} / \mathrm{Hz}$, which represent SE gains of $14.38 \%$ and $6.50 \%$, respectively.

The optimal energy and SINR profiles are analyzed in the sequel for low (Section V-B) and high (Section V-C) activity loads in a very short packet scenario under $n=100$ symbols.

\section{B. Low Traffic Activity Analysis}

The optimized energy profiles $E_{\mathrm{r}}^{1}(t)$ are depicted in Fig. 3 for two representative low traffic loads. When optimizing a non-iterative SIC it is favorable to distribute user-energies exponentially (dashed) whereby stronger users experience higher SINRs [13]. The energy unbalance defined as the ratio between energies of the first and the last users is 5.71 at $\alpha_{1}=0.50$ (dashed black) and 7.50 at $\alpha_{1}=0.70$ (dashed red). When a second decoding attempt is provided to users that fail in the first iteration, it is preferable to design energy distributions with much less unbalance, viz: 1.05 at $\alpha_{1}=0.50$ (solid black) and 1.42 at $\alpha_{1}=0.70$ (solid red).

We now focus on the two-iteration SIC and depict the obtained SINR profiles in the first and the second SIC iterations

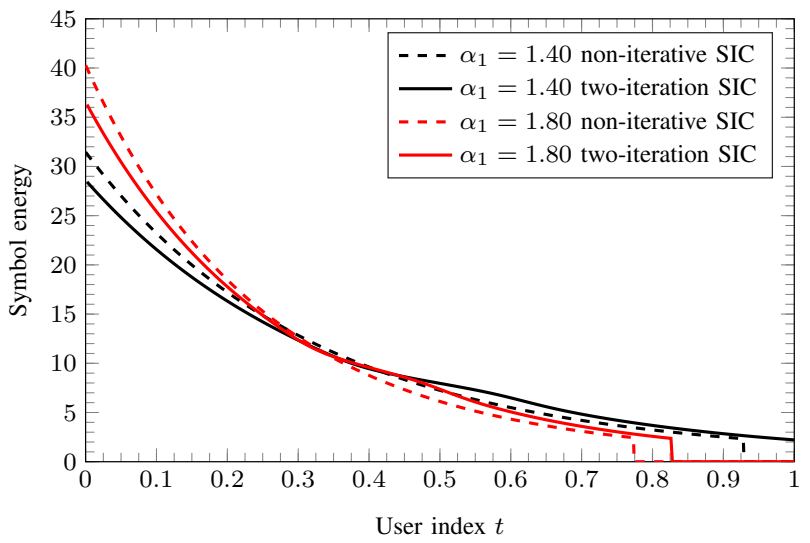

Fig. 5. Optimum energy profile $E_{\mathrm{r}}^{1}(t)$ when optimizing up to the first (dashed) and second (solid) SIC iterations.

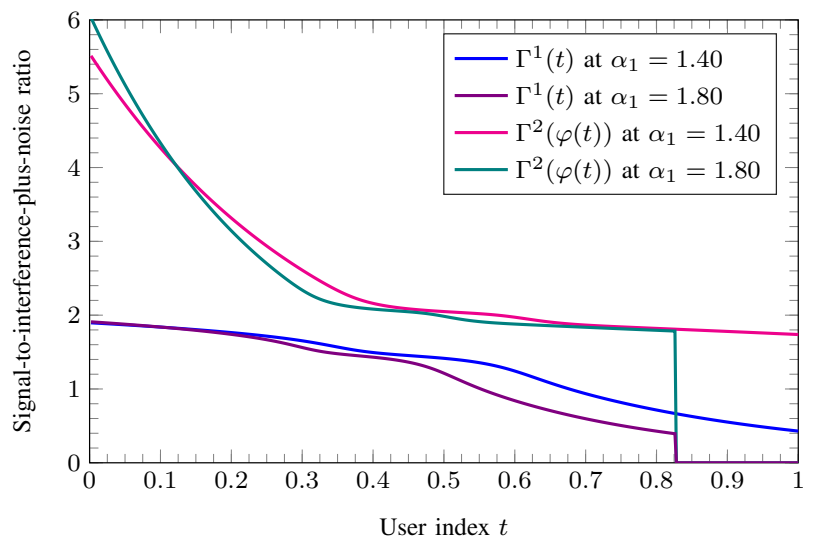

Fig. 6. SINR profiles associated with the first $\Gamma^{1}(t)$ and second $\Gamma^{2}(\varphi(t))$ iterations.

in Fig. 4. As shown, the former energy distributions generate SINR profiles in the first iteration (blue, violet) benefiting the last users of SIC decoding order, and practically uniform SINR profiles in the second iteration (magenta, teal). When the amount of MA interference is low, the former turns out the best to maximize user-SINR gains between iterations.

\section{High Traffic Activity Analysis}

The optimum energy profiles $E_{\mathrm{r}}^{1}(t)$ are depicted in Fig. 5 for the high traffic loads 1.40 and 1.80 . When a non-iterative SIC is adopted, the high amount of MA interference entails the activation of user admission $t_{*}<1$ so as to regulate the admitted load (dashed). When optimizing a two-iteration SIC, higher system load is allowed before $t_{*} \leq 1$ is activated. Specifically, at $\alpha_{1}=1.40$ (solid black) $t_{*}=1$ does not enable load regulation, and a user-energy distribution close to exponential with less energy unbalance optimizes the SE. The average PER (24) is 0.02. $t_{*} \leq 1$ is enabled with a higher value than that $t_{*}$ associated with a non-iterative SIC because the two-iteration SIC admits higher traffic.

As shown in Fig. 6, the former profiles generate decreasing SINR profiles with higher SINR unbalance as $\alpha_{1}$ increases. In particular, stronger users increase their individual performances in detriment to those associated with weak users. 


\section{CONCLUSION}

We have optimized a SIC demodulator performing two peruser iterations in the large user regime. A bijection mapping is introduced to relate users that remain undecoded between iterations, based on univariate and bivariate PER functions that model non-ideal decoding in the first and the second iterations. Variational calculus is used to optimize the spectral efficiency within an applicable average energy constraint. Comparative analyses under the best $n$-blocklength decoders have shown that the performance of a two-iteration SIC substantially outperforms that of a non-iterative SIC when using very short packet lengths and moderate average energies. The optimum energy allocation is less unbalanced than that under a noniterative SIC. For low activity loads, users achieve almost equal SINRs, whereas for high loads stronger users increase considerably their SINRs in detriment of weak users' SINRs.

\section{APPENDIX}

This appendix tackles the optimization problem (26)-(28). The Lagrangian $\mathcal{L}$ that incorporates constraints (27)-(28) using the scalar $\lambda$ and function $\lambda \beta(t)$, respectively, is

$$
\begin{aligned}
\mathcal{L} & \triangleq \int_{0}^{t_{*}} \operatorname{PER}\left[\Gamma^{2}(\varphi(t))\right] \mathrm{d} t+\lambda\left(\int_{0}^{t_{*}} \Gamma^{1}(t) N_{t}^{1}(t) \mathrm{d} t-\bar{E}\right) \\
& +\lambda \int_{0}^{t_{*}} \beta(t)\left[\Gamma^{1}(t) N_{t}^{1}(t)-\Gamma^{2}(\varphi(t)) N_{t}^{2}(\varphi(t))\right] \mathrm{d} t . \quad(31)
\end{aligned}
$$

As typically considered, variations $\Gamma^{1}(t)=\Gamma_{0}^{1}(t)+a \cdot \phi(t)$, and $\Gamma^{2}(\varphi(t))=\Gamma_{0}^{2}(\varphi(t))+b \cdot \psi(t)$ are taken over the sought profiles. $a, b$ are their magnitudes, and $\phi(t), \psi(t)$ their directions.

For the sake of brevity, we define the auxiliary functions $I(t) \triangleq \int_{t}^{t_{*}} E_{\mathrm{r}}^{1}(\tau) \mathrm{d} \tau$ and $I_{\mathrm{x}}(t) \triangleq \int_{t}^{t_{*}} \beta(\tau) E_{\mathrm{r}}^{1}(\tau) \mathrm{d} \tau$. Moreover, the explicit argument $t$ of all variables is temporarily suppressed to simplify notation. Now, one stationary point equation for each variation is computed in subsections A and B.

\section{A. Stationary Point Equation over $\Gamma^{1}(t)$}

The last three terms depend on $\Gamma^{1}$. The limit of the gradient $\nabla_{a} \mathcal{L}$ at $a \rightarrow 0$ is set to zero. We compute it in two steps:

1) We first group the second and third terms of (31) getting $\lambda \int_{0}^{t_{*}}(1+\beta) \Gamma^{1} N_{t}^{1} \mathrm{~d} t$, where both $\Gamma^{1}$ and $N_{t}^{1}$ depend on $a$. After applying the product differentiation rule and integrating by parts, the following is obtained

$$
\lambda \int_{0}^{t_{*}}\left[(1+\beta) N_{t}^{1}-\alpha_{1}\left(I+I_{\mathrm{x}}\right) \Phi^{\prime}\right] \phi \mathrm{d} t .
$$

2) We compute the gradient of $-\lambda \int \beta \Gamma^{2} N_{t}^{2} \mathrm{~d} t$ where $N_{t}^{2}=N_{t}^{1}\left(t_{*}\right) \exp \left(-\alpha_{1} \int_{0}^{t} \Psi \mathrm{d} \tau\right)$ depends on $\Gamma^{1}$ through $\Psi$ and $N_{t}^{1}\left(t_{*}\right)$. Hence, the gradient is computed, first applying the product differentiation rule, followed by integration by parts:

$$
\alpha_{1} \lambda \int_{0}^{t_{*}}\left[I_{\mathrm{x}}(0) \Phi^{\prime}+I_{\mathrm{x}} \Psi_{\Gamma^{1}}\right] \phi \mathrm{d} t .
$$

The complete gradient is obtained by adding (32)-(33):

$$
\nabla_{a \rightarrow 0} \mathcal{L}=\lambda \int_{0}^{t_{*}}\left[(1+\beta) N_{t}^{1}-\alpha_{1} \tilde{I} \Phi^{\prime}+\alpha_{1} I_{\mathrm{x}} \Psi_{\Gamma^{1}}\right] \phi \mathrm{d} t,
$$

with $\tilde{I} \triangleq I+I_{\mathrm{x}}-I_{\mathrm{x}}(0)=\int_{t}^{t_{*}} E_{\mathrm{r}}^{1}(\tau) \mathrm{d} \tau+\int_{0}^{t} \beta(\tau) E_{\mathrm{r}}^{1}(\tau) \mathrm{d} \tau$.
The stationary point equation is found when (33) vanishes for all admissible $\phi$, and, applying the Fundamental Lemma of the Calculus of Variations (FLCV), its integrand must vanish as well. Therefore, we obtain the following in $0 \leq t \leq t_{*}$

$$
(1+\beta) N_{t}^{1}-\alpha_{1} \tilde{I} \Phi^{\prime}\left[\Gamma_{0}^{1}\right]+\alpha_{1} I_{\mathrm{x}} \Psi_{\Gamma^{1}}\left[\Gamma_{0}^{1}, \Gamma_{0}^{2}\right]=0 .
$$

\section{B. Stationary Point Equation over $\Gamma^{2}(\varphi(t))$}

Secondly, $\nabla_{b \rightarrow 0} \mathcal{L}$ is set to zero. We differentiate the first term of (31) getting $\int_{0}^{t_{*}} \mathrm{PER}^{\prime}\left[\Gamma_{0}^{2}\right] \psi \mathrm{d} t$, and after applying the product differentiation rule to $-\lambda \int_{0}^{t_{*}} \beta \Gamma^{2} N_{t}^{2} \mathrm{~d} t$, we get

$$
-\lambda \int_{0}^{t_{*}}\left(\beta N_{t}^{2} \psi-\alpha_{1} \beta E_{\mathrm{r}}^{1} \int_{0}^{t} \Psi_{\Gamma^{2}} \psi \mathrm{d} \tau\right) \mathrm{d} t .
$$

The right term is integrated by parts with $u=\alpha_{1} \int_{0}^{t} \Psi_{\Gamma^{2}} \psi \mathrm{d} \tau$ and $\mathrm{d} v=\beta E_{\mathrm{r}}^{1} \mathrm{~d} t$. Substituting results into (36) we obtain

$$
\nabla_{b \rightarrow 0} \mathcal{L}=\int_{0}^{t_{*}}\left[\operatorname{PER}^{\prime}\left[\Gamma_{0}^{2}\right]-\lambda\left(\beta N_{t}^{2}-\alpha_{1} I_{\mathrm{x}} \Psi_{\Gamma^{2}}\right)\right] \psi \mathrm{d} t
$$

Now, making use of the FLCV, we finally get

$$
\operatorname{PER}^{\prime}\left[\Gamma_{0}^{2}\right]=\lambda\left(\beta N_{t}^{2}-\alpha I_{\mathrm{x}} \Psi_{\Gamma^{2}}\left[\Gamma_{0}^{1}, \Gamma_{0}^{2}\right]\right) .
$$

\section{REFERENCES}

[1] H. Zhang, S. Chen, X. Li, H. Ji, and X. Du, "Interference management for heterogeneous networks with spectral efficiency improvement," IEEE Wireless Commun., vol. 22, no. 2, pp. 101-107, Apr. 2015.

[2] L. Dai, B. Wang, Z. Ding, Z. Wang, S. Chen, and L. Hanzo, "A survey of non-orthogonal multiple access for 5G," IEEE Commun. Surveys Tuts., vol. 20, no. 3, pp. 2294-2323, thirdquarter 2018.

[3] S. Hu and W. Chen, "Successive amplify-and-forward relaying with network interference cancellation," IEEE Trans. Wireless Commun., vol. 17, no. 10, pp. 6871-6886, Oct. 2018.

[4] T. M. Cover and J. A. Thomas, Elements of information theory. John Wiley \& Sons, 2012.

[5] X. Zhang and M. Haenggi, "The performance of successive interference cancellation in random wireless networks," IEEE Trans. Inf. Theory, vol. 60, no. 10, pp. 6368-6388, Oct. 2014.

[6] G. Durisi, T. Koch, and P. Popovski, "Toward massive, ultrareliable, and low-latency wireless communication with short packets," Proc. IEEE, vol. 104, no. 9, pp. 1711-1726, Sep. 2016.

[7] F. Collard and R. De Gaudenzi, "On the optimum packet power distribution for spread ALOHA packet detectors with iterative successive interference cancelation," IEEE Trans. Wireless Commun., vol. 13, no. 12, pp. 6783-6794, Dec. 2014.

[8] F. Molina and J. Sala-Álvarez, "Average PER performance metrics of iterative successive interference cancellation," IEEE Wireless Commun. Lett., vol. 9, no. 1, pp. 74-77, Jan. 2020.

[9] C. S. Park and K. B. Lee, "Transmit power allocation for successive interference cancellation in multicode MIMO systems," IEEE Trans. Commun., vol. 56, no. 12, pp. 2200-2213, Dec. 2008.

[10] M. Zeng, A. Yadav, O. A. Dobre, and V. Poor, "Energy-efficient joint user-RB association and power allocation for uplink hybrid NOMAOMA," IEEE Internet Things J., vol. 6, no. 3, pp. 5119-5131, Jun 2019.

[11] S. Verdú and S. Shamai (Shitz), "Spectral efficiency of CDMA with random spreading," IEEE Trans. Inf. Theory, vol. 45, no. 2, pp. 622640, Mar. 1999.

[12] D. V. Djonin and V. K. Bhargava, "Asymptotic analysis of the conventional decision feedback receiver in fading channels," IEEE Trans. Wireless Commun., vol. 2, no. 5, pp. 1066-1078, Sep. 2003.

[13] J. Sala-Álvarez, F. Rey, J. Villares, and F. Molina, "Minimum PER user-energy profile for massive SIC receivers under an average energy constraint," in 2017 IEEE 18th Int. Workshop on Signal Processing Advances in Wireless Communications (SPAWC), Jul. 2017, pp. 1-6.

[14] F. Molina, J. Sala-Álvarez, J. Villares, and F. Rey, "Joint energy and rate allocation for successive interference cancellation in the finite blocklength regime," in 2018 6th IEEE Global Conference on Signal and Information Processing (GlobalSIP), Sep. 2018, pp. 1-5. 